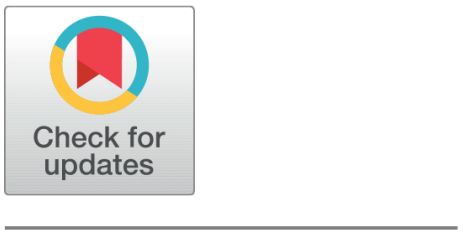

OPEN ACCESS

Received: 12.03 .2021

Accepted: 23.04.2021

Published: 10.05.2021

Citation: Vasumathi MT, Kamarasan M (2021) An Effective Pomegranate Fruit Classification Based On CNN-LSTM Deep Learning Models. Indian Journal of Science and Technology 14(16): 1310-1319. https://doi.org/

10.17485/IJST/V14i16.432

* Corresponding author.

vasumathi_mt@yahoo.com

Funding: None

Competing Interests: None

Copyright: @ 2021 Vasumathi \& Kamarasan. This is an open access article distributed under the terms of the Creative Commons Attribution License, which permits unrestricted use, distribution, and reproduction in any medium, provided the original author and source are credited.

Published By Indian Society for Education and Environment (iSee)

ISSN

Print: 0974-6846

Electronic: 0974-5645

\section{An Effective Pomegranate Fruit Classification Based On CNN-LSTM Deep Learning Models}

\author{
M T Vasumathi ${ }^{1}{ }^{*}$, M Kamarasan $^{2}$ \\ 1 Research Scholar, Department of Computer \& Information Science, Annamalai University, \\ Annamalai Nagar, Chidambaram, Tamilnadu, India \\ 2 Assistant Professor, Department of Computer \& Information Science, Annamalai University, \\ Annamalai Nagar, Chidambaram, Tamilnadu, India
}

\begin{abstract}
Objectives: To employ a deep learning technique that would sort the fruits into normal and abnormal based on the features such as fruit colour, number of fruit spots, and shape of the fruit. Methods: A combined CNN LSTM deep learning model is applied to classify a set of 6519 fruits into two classes namely normal and abnormal. The dataset is an excel file with each record consisting of 9 features. Convolutional Neural Networks (CNN) are applied for deep feature extraction and Long-Short Term Memory (LSTM) is used to detect the class based on extracted features. Findings: The proposed system achieved an accuracy of $98.17 \%$, specificity of $98.65 \%$, sensitivity of $97.77 \%$, and an F1-score of $98.39 \%$. Novelty: The sensitivity of disease detection was less with lesser availability of enhanced detection methods for detecting disease in earlier stages. The issue with these various existing algorithms is that the accuracy was reduced since some sources of errors were not eliminated. Deep Learning delivers methodologies, approaches, and functionalities that can help to resolve analytic and predictive analysis accurately.
\end{abstract}

Keywords: Deep Learning; CNN LSTM; Classification; Hyperparameters

\section{Introduction}

Pomegranate ${ }^{(1)}$ is one of the major fruits produced in India. According to International Trade Centre India stands first in the production of pomegranates worldwide. Approximately 5 percent of the fruits produced in our country are exported every year. Pomegranate export earns a considerable foreign exchange for our country but not much research found to be carried out on pomegranate fruit quality classification. It is therefore essential to classify the fruits into normal and abnormal accurately post-yield given marketing and export. The presence of disease in the fruit can be easily detected by external features like the colour of the fruit, the lesions or black spots, the weight of the fruit, the plant stand and so on. There are several studies carried out to detect diseases in fruits using Convolutional Neural Network Some of the work related to the application of $\mathrm{CNN}$ are given below. 
Tomato leaf disease detection using $\mathrm{CNN}^{(2)}$ employed 3 convolutional layers. The hyperparameters of this model are 10000 epochs each with a batch size of 64 , a learning rate of 0.001 and a dropout rate of 0.5 . This model has generated a testing accuracy of $91.2 \%$. In a VGGNet CNN Model developed to detect diseases in sugarcane ${ }^{(3)}$ having 40 epochs gives a training accuracy of $95.40 \% .{ }^{(4)}$ Classified and graded okra using ResNet50 CNN model and it has produced a classification accuracy of 99.17\%. An attempt to detect diseases in maize using $\mathrm{CNN}^{(5)}$ resulted in a validation accuracy of $99.28 \%$. The deep learning approach in detecting diseases in the fruit guava ${ }^{(6)}$ is carried out by Farhan and Rubaiya shows a testing accuracy of 95.61 . The data size of the system is 10000 and the number of epochs for training the dataset is 50 .

Another CNN LSTM model application for apple disease detection ${ }^{(7)}$ was able to classify good and bad apples with an accuracy of $99.2 \%$. The experimental analysis of the ANN classifier algorithm combined with the SVM classifier algorithm in citrus fruit disease detection ${ }^{(8)}$ has shown a classification accuracy of $88.96 \%$. Plant disease Detection using Deep Learning ${ }^{(9)}$ has achieved a testing accuracy of $98 \%$ with hyperparameters of epoch numbers 25 and a batch size of 32 . The learning rate of the CNN Model in this research is 1e-1. It uses the activation layer Relu. Another research for plant disease detection using tensor flow ${ }^{(10)}$ was able to detect diseases such as bacterial blight, Cercospora, anthracnose with a testing accuracy of $90 \%$.

Similarly in an approach to classify mango leaves infected by anthracnose disease ${ }^{(11)}$ using CNN has made a classification with the accuracy of $97.13 \%$. A deep convolutional neural network training model ${ }^{(12)}$ to identify 14 crop species and 26 diseases achieved an accuracy of $99.35 \%$. Fruit recognition from images using deep learning ${ }^{(13)}$ had recognized different types of fruits such as apple pomegranate etc. with an accuracy of $98 \%$ Fruit recognition using images is carried out using CNN ${ }^{(14)}$ and obtained an accuracy of $100 \%$. There are very few works done in detecting diseases in pomegranate fruit and leaves using deep neural networks and CNN. Some of the related work is listed below. A recent CNN Model developed to detect pomegranate plant leaf disease ${ }^{(15)}$ using $\mathrm{CNN}$ has achieved a $100 \%$ accuracy in classifying a good fruit and obtained an accuracy of $85.71 \%$ in detecting bacterial blight disease. In another work to detect diseases in pomegranate using $\mathrm{CNN}^{(16)}$ where an input of 60 images are given a good fruit detected with $88 \%$ accuracy and an infected fruit detected with an average accuracy of $79 \%$. A work to detect bacterial blight disease in pomegranate fruit ${ }^{(17)}$ using CNN has a classification accuracy of $90 \%$. A multi class SVM ${ }^{(18)}$ with K-means clustering for classification of diseases gives a 90\% of accuracy. ${ }^{(19)}$ Pomegranate fruits are classified as healthy and diseased using the SVM classifier and ANN and it resulted in a classification accuracy of $80 \%$ and $92.65 \%$ respectively.

It can be observed from various researches carried on to detect plant or fruit diseases using deep learning, CNN approach has proven to produce effective accuracy results The present study describes the accuracy obtained from machine-based models to classify pomegranate into two classes normal and abnormal. Healthy fruits are referred to as normal and diseased fruits are referred to as abnormal. The data is collected by observing the important features of fruits that quickly exhibits the quality of fruit and is recorded. Disease prediction in the fruit is connected to many factors such as weight of the fruit, number of spots on the fruit, fruit shape, the plant stand and defoliation in the tree. The classification of pomegranate fruits is carried out by a classifier model that was trained on the training data to predict the class label of new testing data. The novelty that our present work provides is feature extraction task is done using CNN as in previous researches but the LSTM model is combined with CNN to classify fruits which is considerably improving the accuracy of classification. The main advantage of using LSTM for classification is that it uses several parameters such as learning rate, input bias, output bias which minimizes the complexity of weight update.

\section{Related Theory and Fundamentals}

The following section introduces CNN and LSTM used in this study.

\subsection{Convolutional Neural Network (CNN)}

$\mathrm{CNN}^{(20)}$ is a type of deep neural network which mimics a biological neural network. It is suitable for pattern recognition and voice recognition. Being an efficient tool in machine learning it is popular in classification dataset. CNN is ideal for processing $2 \mathrm{D}$ images. $\mathrm{CNN}$ is made up of several layers which include the Input layer, Convolutional layer, pooling layer fully connected layer, ReLu, Drop out and classification layers as shown in Figure 1. The functions of these layers are briefed below.

Input Layer: This is the first layer of CNN. In this layer pre-processed input image is given to the network.

Convolutional Layer: It is the basis of the CNN model. It is responsible for extracting feature maps by using pixel matrices of images. This layer produces a new image matrix or feature map. The operation of the convolution layer is given as

$$
F(i, j)=(I * K)(i, j)=\sum \sum(i+m, j+n) K(m, n)
$$

Where I denote input matrix, $\mathrm{K}$ denotes a 2D filter, F represents the output of a 2D feature map. 
Rectified Linear Unit (ReLu): This layer comes after the convolution layer. It consists of an activation function that will output the input directly if it is positive otherwise it will output zero. This layer is also called as non-linearity layer. It is mathematically expressed as

$$
f(x)=\max (0, x)
$$

Pooling Layer: This layer reduces the dimension of the feature maps generated by the convolutional layer. Therefore, it reduces the number of parameters to learn and the amount of computation.

Fully Connected Layer: This layer summarizes the results of the previous layers and uses them to classify the image into a label. The neurons in this layer are fully connected to all areas of the previous layer.

Dropout Layer: This layer helps prevent the model from over fitting. Dropout can be implemented in any layers but not in the output layer. This layer uses a hyperparameter called dropout that randomly sets the outgoing edges of hidden units to 0 at each update of the training phase. This layer is responsible for the random deletion of some nodes in the network.

Classification Layer: This layer comes after the fully connected layer and here is where the classification takes place.

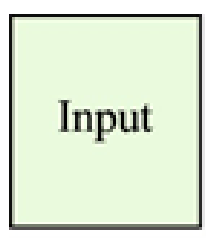

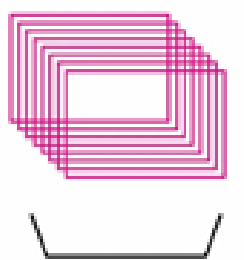

Convolution w/ReLU

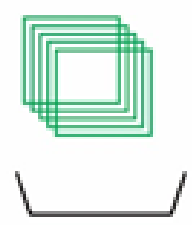

Pooling

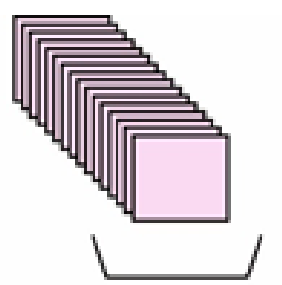

Fully Connected Fully Connected w/ReLU

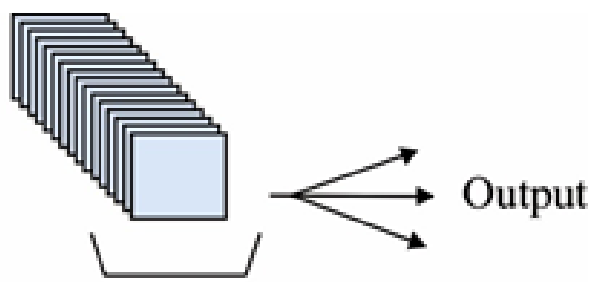

Fig 1. CNN Model

\subsection{Long Short Term Memory (LSTM)}

Long Short-Term Memory network, or LSTM ${ }^{(21)}$ for short, is a category under recurrent neural networks that provides stateof-the-art results on several complicated prediction problems. It is used to solve the problem of short term memory using gates which helps in the proper flow of information. The long term memory in LSTM is called cell state and the cell state is modified by three gates namely input gate, forget gate and output gate.

The architecture of the LSTM model is shown in Figure 2.

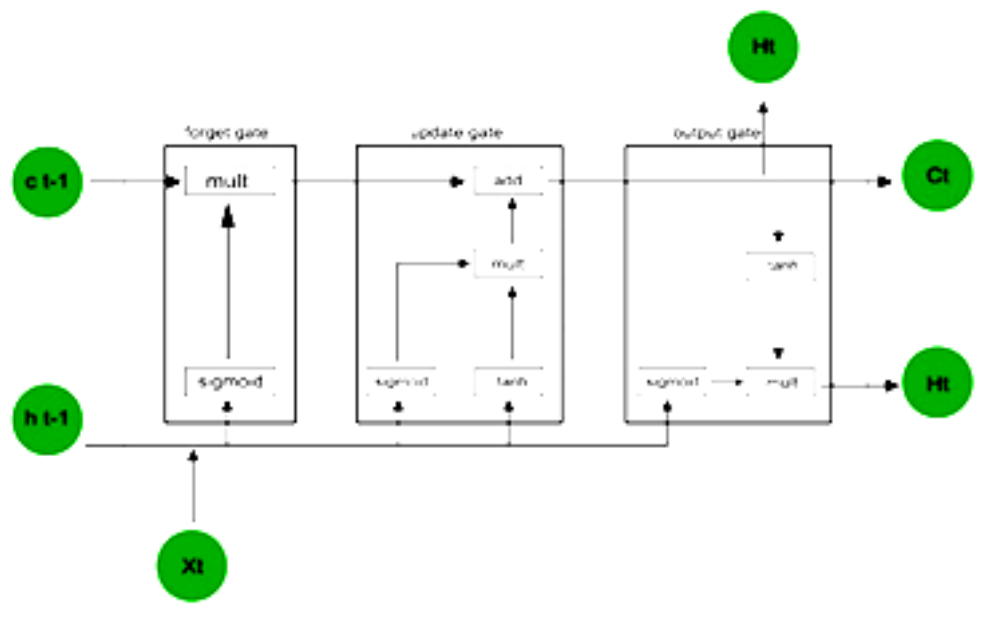

Fig 2. LSTM Model 
These gates function as filters and their purpose is outlined as follows.

Input Gate: This gate is responsible to decide which new information should be added to the LSTM cell state given the previous hidden state and new input data.

The principle of the input gate of LSTM is described below

$$
\begin{aligned}
& \mathrm{i}_{\mathrm{t}}=\sigma\left(\mathrm{W}_{\mathrm{i}} \cdot\left[\mathrm{h}_{\mathrm{t}-1}, \mathrm{X}_{\mathrm{t}}\right]+\mathrm{b}_{\mathrm{i}}\right) \\
& \widetilde{C}_{t}=\tanh \left(\mathrm{W}_{\mathrm{i} \cdot} \cdot\left[\mathrm{h}_{\mathrm{t}-1}, \mathrm{x}_{\mathrm{t}}\right]+\mathrm{b}_{\mathrm{i}}\right) \\
& \mathrm{C}_{t}=f_{t} C_{t-1}+i_{t} \widetilde{C}_{t}
\end{aligned}
$$

Where, $\mathrm{W}_{i}$ denotes weight matrices

$\mathrm{b}_{i}$ denotes input gate bias

$\mathrm{C}_{t}=$ memory information

$\widetilde{C}_{t}=$ tanh output

Forget Gate: This gate decides which bits of the cell state are useful given both the previous hidden state and new input data.

The principle of the output gate is given below

$$
f_{t=} \sigma\left(W_{f} \cdot\left[h_{t-1}, x_{t}\right]+b_{f}\right.
$$

Where, $W_{f}$ represents the weight matrix

$\mathrm{b}_{f}$ is the offset

$\sigma$ is the sigmoid function

Output Gate: The output gate decides the new hidden state based on the newly updated cell state, previously hidden state and the new input data. The output gate principle is shown below

$$
\begin{gathered}
O_{t}=\sigma\left(W_{o} \cdot\left[h_{t-1}, x_{t}\right]+b_{o}\right. \\
\mathrm{h}_{\mathrm{t}}=\mathrm{O}_{\mathrm{t}} \tanh \left(\mathrm{C}_{\mathrm{t}}\right)
\end{gathered}
$$

where $W_{o}$ denote output gate's weighted matrices

$b_{o}=$ LSTM bias

\subsection{CNN-LSTM Model}

The CNN model described above is capable of handling a single image transforming it from input pixels to a vector representation. But we need to repeat this process for multiple images and allow the LSTM to build up an internal state by updating weights. Hence our proposed work uses a single CNN model and a sequence of LSTM models one for each time step. Here CNN Model is applied to each input image and the output is input to the LSTM as a single time step.

\section{Proposed Methodology}

In the current study a deep model is proposed that is based on deep features extracted using CNN and LSTM network. The deep features are extracted from fully connected layers. The extracted deep features are sent as input to the LSTM layer. After LSTM layer a fully connected layer, a softmax layer and a classification layer are used that would sort the images to normal and abnormal which are represented with class labels 0 and 1 respectively. In the current study we extracted deep features by samples to work through before updating the deep network parameters.

The structure of the proposed work is shown in Figure 3 


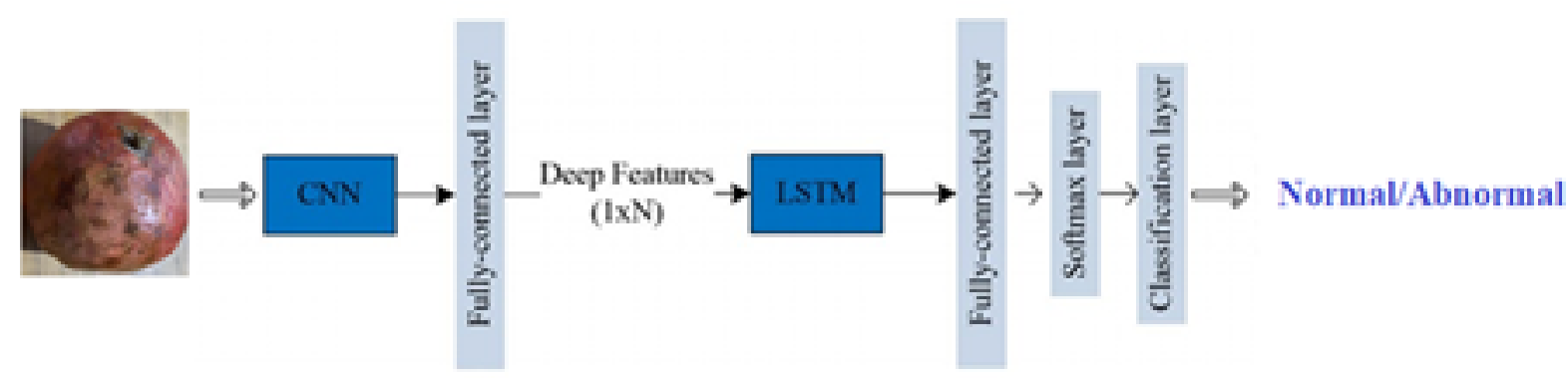

Fig 3. Proposed CNN LSTM Framework

\section{Result and Discussions}

This section describes the dataset, experimental works and the results achieved in detail.

\subsection{Dataset Acquisition}

Due to the non-availability of the benchmark dataset on pomegranate fruits, we have obtained the data by visiting pomegranate farms in and around Bangalore. Twelve farms are visited and images of fruits are captured using the DSLR and iPhone camera. The images were captured in the daylight. Some images are downloaded from Kaggle Dataset and Cofilab Dataset. Some representative image samples from the dataset are shown in Figure 4. As given in Table 1 the dataset includes a total of 6519 images belonging to two different classes namely healthy and diseased. The dataset contains normal and abnormal fruits in the ratio $2: 1$ respectively which is a balanced dataset.
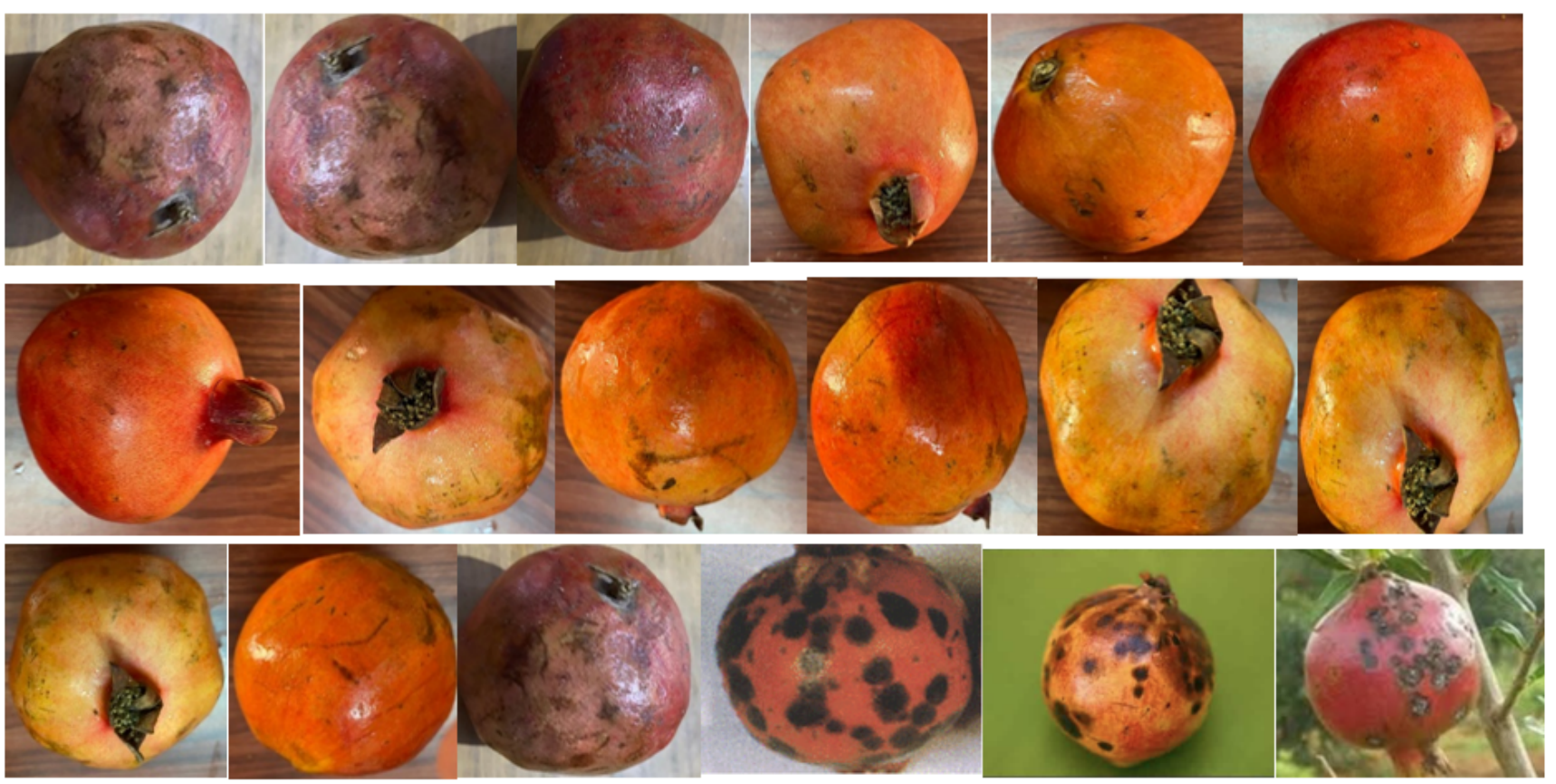

Fig 4. Samples of Pomegranate normal and abnormal images 
Table 1. Dataset Details

\begin{tabular}{lll}
\hline Label & Name & Number of Images \\
\hline 0 & Abnormal & 2813 \\
1 & Normal & 3706 \\
\hline
\end{tabular}

\subsection{Experimental Setup}

The proposed model was implemented using the Python toolbox. The experiment was conducted using a MacBook pro with an 8-core CPU with 4 performance cores and 4 efficiency cores,8-core GPU,16-Neural engine and 8GB of unified memory. During the experiments, $62 \%$ of the dataset was used for training and the remaining $38 \%$ used for testing the proposed model.

\subsubsection{Significance of hyperparameters}

Hyperparameters ${ }^{(22)}$ are those that directly control the behaviour pattern of the training Algorithm and have a significant impact on the performance of the model being trained. A CNN Model with finely tuned hyperparameters can yield the best results. The most important hyperparameters are the number of epochs, learning rate and drop out.

\section{Number of Epochs}

An epoch ${ }^{(23)}$ represents the number of passes taken by the machine learning algorithm to complete the entire training dataset. Datasets are usually grouped into batches when the amount of data is very large. If the batch size is the whole training dataset then the number of epochs is the number of iterations. The number of epochs needed for a model to run is decided based on several parameters that are related to both the dataset used and the purpose of the model, and where there have been efforts to turn this process into an algorithm, often a deep understanding of the data itself is indispensable. The number of epochs used in our model is 50 .

\section{Learning Rate}

The magnitude of the weights is continuously updated during the training. This process of weight update is called learningrate $^{(24)}$. Thus, learning-rate is a hyperparameter used in the training of neural networks and its value ranges between 0.0 and 1.0. The learning rate of the current model is 0.001 .

\section{Activation Function}

ReLU is an acronym of Rectified Linear Unit and is a kind of activation function ${ }^{(25)}$. Mathematically, it is expressed as $\mathrm{Y}=$ $\max (0, \mathrm{x})$. Visually, it looks as in Figure 5. ReLU is the most commonly used activation function in neural networks, especially in CNNs. It will output the input directly if it is positive, otherwise, it will output zero. The ReLu activation function enables models to learn quickly and perform better by overcoming the problem of vanishing gradient.

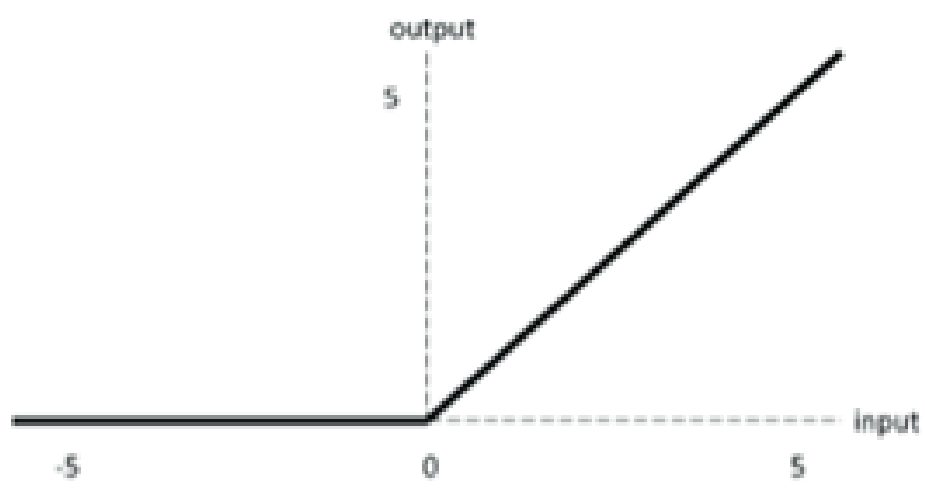

Fig 5. Graphical representation of ReLu 


\section{Dropout Rate}

Dropout ${ }^{(26)}$ is a process that some randomly chosen neurons are ignored during training. The dropped out neurons implies that their contribution to the activation of downstream neurons is temporarily eliminated on the forward pass and no weight updates happen to the neurons on the backward pass.

The current CNN Model hyperparameter values are shown below Table 2

\begin{tabular}{ll} 
& Table 2. Model Training Parameters \\
\hline Hyperparameter & Value \\
\hline Epochs & 50 \\
Batch size & 114 \\
Learning Rate & 0.001 \\
Activation Function & $\mathrm{ReLu}$ \\
Dropout Rate & 0.5 \\
\hline
\end{tabular}

\subsubsection{Performance Evaluation}

The confusion matrix ${ }^{(27)}$ or error matrix is an error specific table that shows the performance of the proposed model. It can be a $2 \mathrm{X} 2$ table for binary classification or a table with more dimensions depending on multiway classification. Our work uses a binary classifier to predict whether a fruit has a disease or not. It consists of two rows and columns where it consists of values as follows.

Normal- Absence of diseases; Abnormal- Presence of diseases

True positive (TP)- No. Of samples correctly identified as abnormal

False positive (FP)- No. Of samples incorrectly identified as abnormal

True Negative (TN)- No. Of samples correctly identified as normal

False Negative (FN)- No. Of samples incorrectly identified as normal.

Sensitivity: It is defined as the proportion of real positive cases that are correctly predicted positive. It is given as:

$$
\text { Sensitivity }=\frac{T P}{T P+F N}
$$

Specificity: It is represented as the proportion of actual negative, which got predicted as the negative. It can be represented as

$$
\text { Specificity }=\frac{T N}{T N+F P}
$$

Recall: It indicates the proportion of actual positives which are identified correctly. It can be determined by

$$
\text { Recall }=\frac{T P}{T P+F N}
$$

F-score: It is a measure of model accuracy on the applied dataset. It can be equated as follows.

$$
F-\text { score }=2 * \frac{\text { precision } * \text { recall }}{\text { precision }+ \text { recall }}
$$

Precision: It is defined as the proportion of predicted positives that are correctly real positives. It is given as:

$$
\text { Precision }=\frac{\mathrm{TN}}{\mathrm{TN}+\mathrm{FP}}
$$

Accuracy: It is the most institutional performance that measures the correctly predicted observation to the total observations. It defines the ability to distinguish between normal and abnormal cases. It is given as:

$$
\text { Accuracy }=\frac{T P+T N}{T P+T N+F P+F N}
$$

False positives and false negatives correspond to type-I and type II error respectively. The confusion matrix obtained for our model is shown in Figure 6. 


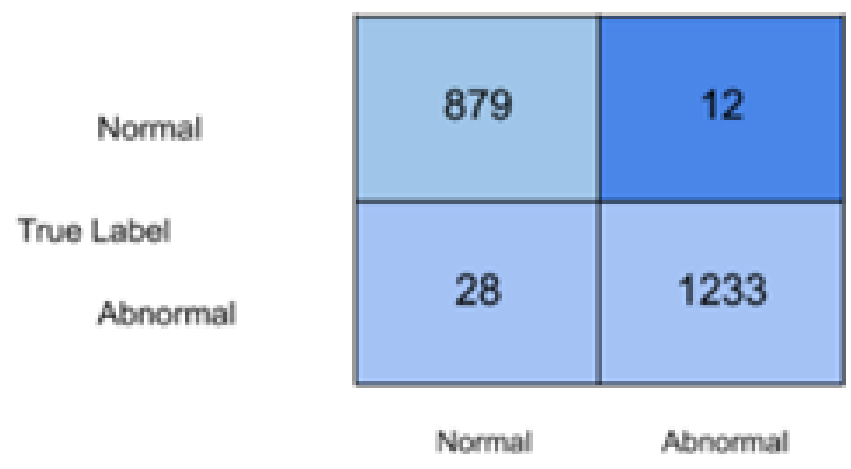

\section{Predicted Label}

Fig 6. Confusion Matrix

The performance of the model is further explored by the computation of precision, recall and F-score. These values are shown in the classification report Table 3. The accuracy of the model is obtained as $98 \%$.

Table 3. Classification Report

\begin{tabular}{|c|c|c|c|c|c|}
\hline & & Precision & Recall & F1-Score & Support \\
\hline & 0 & 0.99 & 0.99 & 0.98 & 891 \\
\hline & 1 & 0.99 & 0.97 & 0.98 & 1261 \\
\hline accuracy & & & & 0.98 & 2152 \\
\hline Macro Avg & & 0.99 & 0.98 & 0.98 & 2152 \\
\hline Weighted Avg & & 0.99 & 0.98 & 0.98 & 2152 \\
\hline
\end{tabular}

\section{Accuracy Graph}

Figure 7 shows the accuracy curve for the given model. The model loss curve is shown in Figure 8.

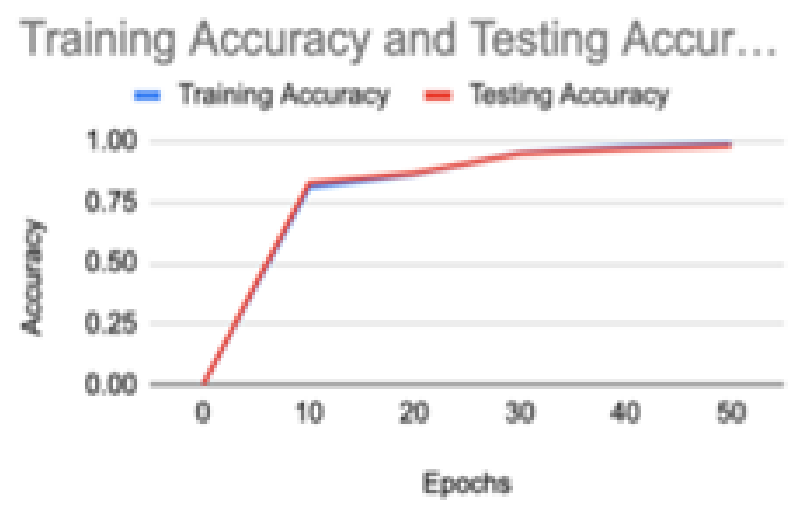

Fig 7. Accuracy comparison curves for training and testing dataset 


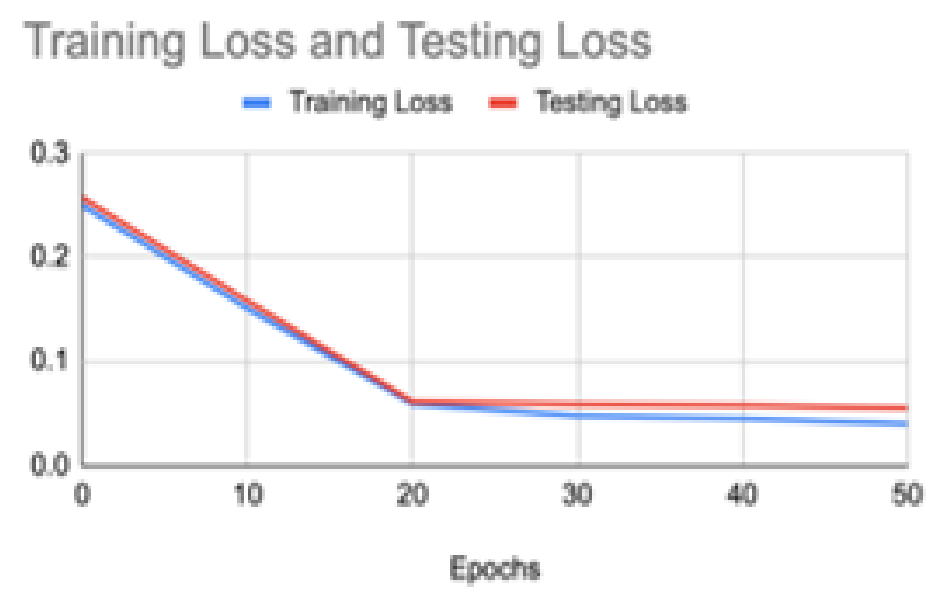

Fig 8. Loss comparison curve of training and testing dataset

In Table 4 we have shown a comparative analysis of our work with previous research carried out in fruit quality classification using machine learning algorithms. It can be observed from the comparison table that CNN LSTM produces more accuracy in the classification process and also it can be noted that considering more data size and trials results in higher accuracy in assessing the quality of fruits and classifying it as normal and abnormal.

Table 4. Comparative Analysis of the Proposed Model with the Existing Research

\begin{tabular}{llll}
\hline SNO & Research Study & Classifier & Accuracy \\
\hline 1 & Current Study & CNN LSTM & 98.17 \\
2 & Pomegranate Disease Classification. ${ }^{(28)}$ & Ada Boost Ensemble Algorithm & 92.9 \\
3 & Diagnosis of Pomegranate Plant diseases $^{(29)}$ & Back Propogation Algorithm & 90 \\
4 & Pomegranate Quality Assessment $^{(30)}$ & SVM & 76.483 \\
5 & Pomegranate fruit quality assessment $^{(19)}$ & ANN & 92.65 \\
\hline
\end{tabular}

\section{Conclusion and Future Work}

The current study implements a CNN LSTM model using Python to detect the diseased pomegranate and classify them into normal and abnormal. The fruit classification accuracy was found to be $98.17 \%$. This accuracy is considerably high compared to the accuracy obtained using the classifiers such as SVM, ANN and Back Propagation Algorithm. But the proposed model is limited with only two classes so as for future enhancement of this research is concerned, we aim to develop a CNN LSTM model with multi-way classification to predict four common pomegranate diseases such as anthracnose, bacterial blight, Cercospora fruit spot and heart rot.

\section{References}

1) Kaur R, Kaushal S. Antimicrobial and antioxidant potential of pomegranate (Punica granatum L.) peel. International Journal of Chemical Studies . 2018;3441(3449).

2) Prajwal TM, Pranathi A, SaiAshritha K, Chittaragi NB, Koolagudi SG. Tomato Leaf Disease Detection Using Convolutional Neural Networks. In: and others, editor. 2018 Eleventh International Conference on Contemporary Computing (IC3). Noida. 2018;p. 1-5. doi:10.1109/IC3.2018.8530532.

3) Militante SV, Gerardo BD. Detecting Sugarcane Diseases through Adaptive Deep Learning Models of Convolutional Neural Network. In: and others, editor. 2019 IEEE 6th International Conference on Engineering Technologies and Applied Sciences (ICETAS). 2019;p. 1-5. doi:10.1109/ICETAS48360.2019.9117332.

4) MRaikar M, M M, ChaitraKuchanur, ShantalaGirraddi, PratikshaBenagi. Classification and Grading of Okra-ladies finger using Deep Learning. Procedia Computer Science. 2020;171:2380-2389. Available from: https://doi.org/10.1016/j.procs.2020.04.258.

5) Sheikh MH, Mim TT, Reza MS, Rabby ASA, Hossain SA. Detection of Maize and Peach Leaf diseases using Image Processing. In: and others, editor. 2019 10th International Conference on Computing, Communication and Networking Technologies (ICCCNT). Kanpur, India. 2019;p. 1-7. doi:10.1109/ICCCNT45670.2019.8944530. 
6) Haque ASMFA, Hafiz R, Hakim MA, Islam GMR. A Computer Vision System for Guava Disease Detection and Recommend Curative Solution Using Deep Learning Approach. In: and others, editor. 2019 22nd International Conference on Computer and Information Technology (ICCIT). Dhaka, Bangladesh. 2019;p. 1-6. doi:10.1109/ICCIT48885.2019.9038598.

7) Turkoglu M, Hanbay D, Sengur A. Multi-model LSTM-based convolutional neural networks for detection of apple diseases and pests. Journal of Ambient Intelligence and Humanized Computing. 2019. Available from: https://doi.org/10.1007/s12652-019-01591-w.

8) Doh B, Zhang D, Shen Y, Hussain F, Doh RF, Ayepah K. Automatic Citrus Fruit Disease Detection by Phenotyping Using Machine Learning. In: and others, editor. 2019 25th International Conference on Automation and Computing (ICAC). 2019;p. 1-5.

9) Ramesh S, Hebbar R, M N, R P, N PB, N S. Plant Disease Detection Using Machine Learning. 2018 International Conference on Design Innovations for 3Cs Compute Communicate Control (ICDI3C). 2018;p. 41-45. doi:10.1109/ICDI3C.2018.00017.

10) Gowda NC, Kumar S, Majumdar S, Abhishek KN, Sarode P. Android Application on Plant Disease Identification using Tensorflow. International Journal of Engineering and Advanced Technology;8(5S).

11) Singh UP, Chouhan SS, Jain S, Jain S. Multilayer Convolution Neural Network for the Classification of Mango Leaves Infected by Anthracnose Disease. IEEE Access. 2019;7:43721-43729. Available from: https://dx.doi.org/10.1109/access.2019.2907383. doi:10.1109/access.2019.2907383.

12) Jadhav SB, Udupi VR, Patil SB. Identification of plant diseases using convolutional neural networks. International Journal of Information Technology. 2020. Available from: https://doi.org/10.1007/s41870-020-00437-5.

13) Mures, an H, Oltean M. Fruit recognition from images using deep learning. Acta Univ Sapientiae, Informatica . 2018;10(1):26-42. doi:arXiv:1712.00580v10.

14) Muhammad NA, Nasir AA, Ibrahim Z, Sabri N. Evaluation of CNN, Alexnet and GoogleNet for Fruit Recognition. Indonesian Journal of Electrical Engineering and Computer Science. 2018;12(2):468-475. Available from: https://dx.doi.org/10.11591/ijeecs.v12.i2.pp468-475.

15) Chakali R. Effective pomegranate plant leaf disease detection using deep learning. International Journal of Circuit, Computing and Networking;1(2):8-10. Available from: https://www.computersciencejournals.com/ijccn/article/13/1-1-14-682.pdf.

16) Manishabhange HA. Hingoli Wala Smart Farming: Pomegranate Disease Detection Using Image Processing. Procedia Computer ScienceVolume 58. 2015;58:280-288.

17) Pawara S, Nawale D, Patil K, Mahajan R. Early Detection of Pomegranate Disease Using Machine Learning and Internet of Things. In: 3rd International Conference for Convergence in Technology (I2CT). 2018;p. 1-4. doi:10.1109/I2CT.2018.8529583.

18) Behera SK, Jena L, Rath AK, Sethy PK. Disease Classification and Grading of Orange Using Machine Learning and Fuzzy Logic. 2018 International Conference on Communication and Signal Processing. 2018;p. 678-682. doi:10.1109/ICCSP.2018.8524415.

19) Dhakate M, B IA. Diagnosis of pomegranate plant diseases using neural network. Fifth National Conference on Computer Vision, Pattern Recognition, Image Processing and Graphics (NCVPRIPG). 2015;p. 1-4. doi:10.1109/NCVPRIPG.2015.7490056.

20) Razak SM, Jafarpour B. Convolutional neural networks (CNN) for feature-based model calibration under uncertain geologic scenarios. Computational Geosciences. 2020;24(4):1625-1649. Available from: https://dx.doi.org/10.1007/s10596-020-09971-4.

21) Kumar J, Goomer R, Singh AK. Long Short Term Memory Recurrent Neural Network (LSTM-RNN) Based Workload Forecasting Model For Cloud Datacenters. Procedia Computer Science. 2018;125:676-682. Available from: https://dx.doi.org/10.1016/j.procs.2017.12.087.

22) Krishnakumari K, Sivasankar E, Radhakrishnan S. Hyperparameter tuning in convolutional neural networks for domain adaptation in sentiment classification (HTCNN-DASC). Soft Computing. 2020;24:3511-3527. Available from: https://dx.doi.org/10.1007/s00500-019-04117-w.

23) Popescu O, Strapparava C. Time corpora: Epochs, opinions and changes. Knowledge-Based Systems. 2014;69:3-13. Available from: https://dx.doi.org/10. 1016/j.knosys.2014.04.029.

24) Yu C, Qi X, Ma H, He X, Wang C, Zhao Y. LLR: Learning learning rates by LSTM for training neural networks. Neurocomputing. 2020;394:41-50. Available from: https://dx.doi.org/10.1016/j.neucom.2020.01.106.

25) Ide H, Kurita T. Improvement of learning for CNN with ReLU activation by sparse regularization. In: 2017 International Joint Conference on Neural Networks (IJCNN). 2017;p. 2684-2691. doi:10.1109/IJCNN.2017.7966185.

26) Li H, Li J, Guan X, Liang B, Lai Y, Luo X. Research on Overfitting of Deep Learning. In: 15th International Conference on Computational Intelligence and Security (CIS). 2019;p. 78-81. doi:10.1109/CIS.2019.00025.

27) Salmon BP, Kleynhans W, Schwegmann CP, Olivier JC. Proper comparison among methods using a confusion matrix. In: and others, editor. 2015 IEEE International Geoscience and Remote Sensing Symposium (IGARSS). Milan. 2015;p. 3057-3060. doi:10.1109/GARSS.2015.7326461.

28) Kantale P, Thakare S. Pomegranate Disease Classification using Ada-Boost Ensemble Algorithm. International Journal of Engineering Research \& Technology. 2020;9(9).

29) Deshpande T, Sengupta S, Raghuvanshi KS. Grading \& identification of disease in pomegranate leaf and fruit. International Journal of Computer Science and Information Technologies. 2014;5(3):4638-4645.

30) R AK, Rajpurohit VS, Jirage BJ. Pomegranate Fruit Quality Assessment Using Machine Intelligence and Wavelet Features. Journal of Horticultural Research. 2018;26(1):53-60. Available from: https://dx.doi.org/10.2478/johr-2018-0006. 